NASA-CR-199348

\title{
Termination shock response to large-scale solar wind fluctuations
}

\author{
R. S. Steinolfson \\ Department of Space Sciences. Southwest Research Institute. San Antonio. Texas
}

\begin{abstract}
The analysis of data recorded by the Voyager 2 spacecraft indicates the presence of large-scale fluctuations in the solar wind ram pressure on the time scale of tens of days. The amplitude of the fluctuations is highly variable but often lies within a factor of 5 to 10 change from an average or mean value of the ram pressure. Since the spacecraft has presumably not encountered the termination shock yet, these fluctuations should eventually interact with the shock and thereby play a role in determining the shock location. Numerical solutions of the time-dependent gasdynamic equations are used to simulate the response of the termination shock to fluctuations in the solar wind ram pressure comparable to those observed. The primary result of this study is that the maximum shock excursion due to the fluctuations is of the order of $1 \mathrm{AU}$, which is much smaller than that predicted by other studies. Additional simulations show that the limited movement is due to the fact that the time scale for the termination shock response is substantially larger than the time scale of the fluctuations. It is also shown that the heliopause acts as a barrier for the fluctuations and confines them to the heliosphere.
\end{abstract}

\section{Introduction}

As the solar wind plasma flows outward from the Sun, it eventually interacts with the surrounding interstellar plasma, and the two plasmas relax to a dynamic pressure equilibrium. The formation of a cavity in the interstellar medium by the solar wind plasma was initially suggested by Davis [1955]. For a pressure equilibrium between the solar and interstellar plasmas to be reached, the solar wind makes a transition from supersonic to subsonic flow at a termination shock [Parker, 1961]. The termination shock probably occurs within the range of 50 to 100 astronomical units $(A U)$ from the Sun [e.g., Suess, 1990]. At some distance beyond the termination shock, the solar plasma interacts directly with the interstellar plasma at an interface separating the two plasmas. This intertace is referred to as the heliopause, and the region containing solar plasma is referred to as the heliosphere.

It is generally believed that the solar system travels through the interstellar medium at a speed of approximately $20 \mathrm{~km} \mathrm{~s}^{-1}$. The thermodynamic conditions of the interstellar medium are not well known, but if the speed of the solar system through this medium is supersonic, a bow shock forms beyond the heliopause to slow and deflect the interstellar plasma. Baranov [1990] has reviewed this two-shock model of the solar wind/interstellar medium interaction. A bow shock would not be expected to form when the speed of the solar system relative to that of the surrounding medium is subsonic.

As summarized in recent reviews on this topic [e.g., Holzer, 1989; Suess, 1990], the general structure of the interaction of the solar wind with the interstellar medium in terms of the bulk or large-scale properties is fairly well understood in a qualitative sense for steady plasma flows. There are, of course, several physical processes of a more detailed or localized nature occurring in the two plasmas that are not well understood themselves (e.g., interstellar neutral

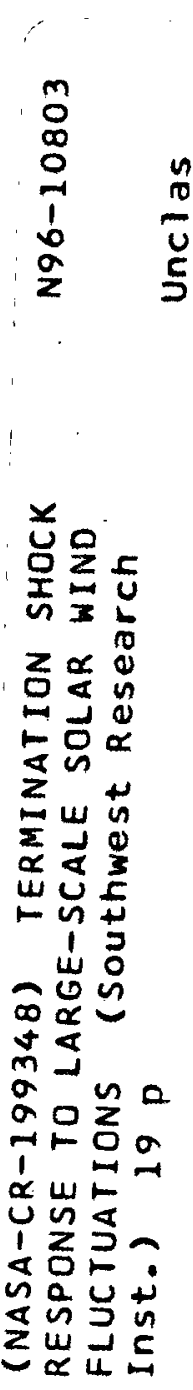


gas, charge exchange, cosmic rays). Their effect on the overall interaction is also not well understood.

The analysis of data from the plasma science experiment on Voyager 2 as the spacecraft traveled from 1 to $40.4 \mathrm{AU}$ indicates that there are large-scale fluctuations in the solar wind ram pressure occurring on the time scale of tens of days [Belcher et al., 1993]. Data from Pioneers 10 and 11 also show the presence of large variations in the ram pressure [Barnes, 1990]. Since the ram pressure plays a major role in controlling the location of the termination shock, it seems logical that large-scale fluctuations in the ram pressure may produce relatively large excursions of the termination shock. Belcher et al. [1993] used a kinematic model in which they limited the termination shock speed to $200 \mathrm{~km} \mathrm{~s}^{-1}$ and estimated that the location may vary by as much as $15 \mathrm{AU}$ in response to the observed ram pressure fluctuations. This excursion amplitude is similar to that computed in other one-dimensional analyses [Barnes, 1993; Suess, 1993]. If there are large-scale movements of the termination shock in response to large-scale fluctuations in the solar wind ram pressure, then a spacecraft that reaches the vicinity of the shock may cross it more than once.

The objective of the present study is to use numerical solutions of the gasdynamic equations to examine the effect that large-scale fluctuations with temporal and spatial scales similar to those observed have on the average or mean behavior of the interaction between the solar wind and interstellar plasmas and particularly on the location of the termination shock. The study is performed for the case of subsonic relative motion between the solar system and the interstellar medium (for reasons discussed in section 3.1). A dynamic equilibrium solution of the interaction is obtained first, and fluctuations in the solar wind ram pressure are then generated to perturb the equilibrium.

\section{Equations and Model}

The gasdynamic equations used in this study can be written in the following nondimensional form:

$$
\begin{gathered}
\frac{\partial \rho}{\partial t}+\nabla \cdot(\rho \mathbf{v})=0, \\
\rho \frac{d \mathbf{v}}{d t}=-\frac{1}{\gamma} \nabla p, \\
\frac{\partial}{\partial t}\left[\frac{1}{2} \rho v^{2}+\frac{p}{\gamma(\gamma-1)}\right]+ \\
\nabla \cdot\left\{\mathbf{v}\left(\frac{1}{2} \rho v^{2}+\frac{p}{\gamma-1}\right)\right\}=0,
\end{gathered}
$$

where $d / d t$ is the total derivative, and the physical variables $\rho, \mathbf{v}$, and $p$ are the density, plasma velocity, and thermal pressure, respectively. The density $\rho=n_{e} / m_{p}$, where $n_{e}$ is the 
electron number density and $m_{p}$ is the proton mass. All variables have been made dimensionless by normalizing the thermodynamic quantities to the initial values at $1 \mathrm{AU}$ and the velocity to the initial sound speed at $1 \mathrm{AU}\left(a_{0}\right)$. Distance is referenced to $1 \mathrm{AU}\left(r_{0}\right)$, and time is normalized by $t_{\mathrm{o}}=r_{\mathrm{o}} / a_{\mathrm{o}}$. The only parameter that enters directly into the above equations is the specific heat ratio $\gamma$, which we take to be $5 / 3$. Note that the potentially important effects of collisions with neutral species, resonance charge exchange, and cosmic ray pressure have not been included. Donohue and Zank [1993] used a gasdynamic model of a localized section of the termination shock to show that acceleration of the anomalous component and/or galactic cosmic rays may significantly influence the nature, structure, and motion of the termination shock. It remains to include the physics of their study into a global model such as that used here. In addition, since this is a gasdynamic model, the magnetic fields of the interstellar medium and of the solar wind are not included. The neglect of the magnetic field in the solar wind should have a minimal effect, since the plasma beta (ratio of thermal pressure to magnetic pressure) is generally large throughout the solar wind. It has been suggested that the magnetic field may provide the largest contribution to the total pressure in the interstellar medium [e.g., Suess, 1990], but the physical nature of the interstellar pressure should not significantly affect the present results.

Equations (1a) - (1c) are solved in the $r-\theta$ plane of a spherical coordinate system aligned such that the pole of the coordinate system at $\theta=0^{\circ}$ points into the direction of motion of the solar system relative to that of the interstellar medium. The solution is independent of the azimuthal angle $\phi$ and thus is axisymmetric about the poles. The coordinate system is centered at the Sun $(r=0)$ and remains fixed with respect to the Sun. Consequently, the relative motion between the solar system and the interstellar medium is represented by a velocity of the interstellar medium $\left(V_{\text {is }}\right)$ in our coordinate system. The simulation box extends from $0^{\circ}$ to $180^{\circ}$ in $\theta$ and from $10 \mathrm{AU}$ to $400 \mathrm{AU}$. The radial grid spacing is constant at $1.4 \mathrm{AU}$, and the angular grid spacing is constant at $1^{\circ}$, which gives a grid of $280 \times 181$.

The equations are solved numerically using a two-step Lax-Wendroff differencing scheme similar to that given by Richtmyer and Morton [1967], with second-order accuracy. As is usual for such schemes, an additional smoothing must be performed on the values from the Lax-Wendroff step in order to remove high-frequency oscillations and the overshoots and undershoots created near ideal discontinuities such as shocks. A smoothing term suggested by Lapidus [1967] was used, as was a flux-corrected-transport term [Book et al., 1975]. The two smoothers produced qualitatively the same results when they both worked. The Lapidus term, though, was considerably more robust in handling the nonuniformities and the substantial range in physical variables in the present problem and was used for the results presented here. The flux-corrected-transport correction required more adjustment of the numerical constants and for some parametric regimes produced questionable results.

An explicit, shock-capturing scheme, as used here, is not 
necessary for computing the initial dynamic equilibrium solution into which the fluctuations are introduced, although it is essential in order to simulate the proper temporal response to the fluctuations. The initial equilibrium could equally well have been computed using a shock-fitting scheme with either an explicit or an implicit method used to solve for the solution between the discontinuities [e.g., Baranov and Malama, 1993]. It might be expected that the addition of a semi-implicit term in the momentum equation would permit the use of a larger time step during the relaxation. However, even with such a term, the time step is still restricted so that plasma cannot be convected farther than a grid spacing in a single time step. With the small grid spacing and the large flow speeds in the solar wind, the time step for a semi-implicit method would be only about $12 \%$ larger that for the explicit method used here. Such a small increase in the time step certainly would not compensate for the additional CPU time necessary to solve the momentum equation implicitly in a semi-implicit method.

Three of the physical variables (radial flow speed $v_{\mathrm{r}}$, density, and pressure) are symmetric at the angular boundaries, while the fourth is antisymmetric (meridional flow speed $v_{\theta}$ ). The supersonic solar wind is specified at $1 \mathrm{AU}$, and an adiabatic atmosphere with constant radial flow speed is used to compute values at the inner computational boundary at $10 \mathrm{AU}$. Since this is a supersonic-inflow boundary, all physical quantities can be specified on it. The interstellar density and velocity are held fixed at the outer radial boundary for $0^{\circ} \leq \theta \leq 90^{\circ}$, and zero-order extrapolation along the local flow direction is used to update the pressure. Since this portion of the outer boundary is a subsonic-inflow boundary, not all of the physical quantities can be specified there. Zero-order extrapolation along the local flow direction is used to obtain all values at the outer radial (subsonicoutflow) boundary for $90^{\circ}<\theta \leq 180^{\circ}$.

The simulation is performed in two separate steps. A dynamic equilibrium is obtained first, and then a fluctuating solar wind flow speed is imposed on this equilibrium at the inner radial boundary. The simulation to compute the dynamic equilibrium is initiated by specifying adiabatic solar wind conditions within 10-40 AU, a termination shock at 40 $\mathrm{AU}$, interstellar conditions beyond $80 \mathrm{AU}$, and a linear fit between the shocked solar wind and the interstellar plasmas. Starting with this nonequilibrium state, the time-dependent simulation continues until the solution relaxes to a dynamic equilibrium. The solar wind fluctuations are then generated in the equilibrium solution by temporal oscillations in the solar wind speed at the inner radial boundary. The thermodynamic conditions at the inner boundary remain unchanged during the flow speed oscillations.

\section{Numerical Results}

\subsection{Dynamic Equilibrium Solution}

The spatial variation of the thermodynamic quantities and the streamlines after the solution has relaxed to a dynamic equilibrium are shown in Figure 1. The physical quantities in the solar wind at $1 \mathrm{AU}$ for this simulation are $v_{\mathrm{r}}=300 \mathrm{~km}$ 
$\mathrm{s}^{-1}, v_{\theta}=0, n_{\mathrm{e}}=3 \mathrm{~cm}^{-3}$, and temperature $T=10^{5 \circ} \mathrm{K}$. The reference sound speed is then $52.45 \mathrm{~km} \mathrm{~s}^{-1}$, and the reference or characteristic time $\left(t_{0}\right)$ is 33.2 days. The fixed, inflow interstellar conditions are $V_{\text {is }}=13.28 \mathrm{~km} \mathrm{~s}^{-1}$ and $n_{\mathrm{e}}=0.2 \mathrm{~cm}^{-3}$. The inflow interstellar temperature is initially set to $10^{40} \mathrm{~K}$ $\left(M_{\mathrm{is}}=0.8\right)$, but this temperature will change slightly as the pressure adjusts at the inflow boundary. The relaxation required 120,000 time cycles, which represents a total physical evolution time of $4.94 \times 10^{5}$ days, or 1353 years. Based on the sound speed in the interstellar medium (16.59 $\mathrm{km} \mathrm{s}^{-1}$ or $3.48 \mathrm{AU} / \mathrm{yr}$ ), this is time for a disturbance to cross the $800 \mathrm{AU}$ numerical box almost six times. This total evolved time should not be regarded as physically relevant; it is simply an indicator of the time required for the solution to adjust to the specified start-up conditions. However, simulated changes in this equilibrium solution due to changes in the boundary conditions should provide realistic estimates of the actual response time of the solar wind/interstellar medium interaction.

The interstellar flow (in our Sun-centered coordinate system) is from left to right in Figure 1. The locations of the termination shock and the heliopause are indicated on the streamline plot. The rapid changes in variables at the termination shock appear as clustered contours on the pressure and temperature plots. The shock does not appear on the density plot because of the linear scaling used for the contours and the fact that the density at the shock is less than the minimum contour. There is a density jump by about a factor of 4 , however, since the termination shock is a strong shock. For this example with subsonic interstellar flow, the termination shock is nearly spherical and does not have the characteristic bullet shape typical of solutions for a supersonic interstellar flow [e.g., Baranov and Malama, 1993; Steinolfson et al., 1994]. The formation of a more spherical termination shock is one reason a subsonic interstellar flow solution is used for the initial state. However, since the present study concentrates on examining the fluctuations along the $0^{\circ}$ pole, the shape of the downstream portion of the termination shock should have virtually no effect on the results.

\subsection{Response to Large-Scale Fluctuations}

A sinusoidal temporal variation in the solar wind radial flow speed is now specified at the inner radial boundary in order to generate fluctuations in the solar wind ram pressure $\left(\rho v^{2}\right)$. The speed oscillation is maintained throughout the numerical computation. Although the change in the boundary conditions occurs only in the flow speed (the thermodynamic quantities are held fixed at the inner boundary), fluctuations in all the physical quantities are generated in the solar wind. The same variation is applied at all angular locations, so the physical situation that is modeled is one in which the flow speed increases and decreases uniformly at all locations on the solar surface. The amplitude of the flow speed oscillation is $100 \mathrm{~km} \mathrm{~s}^{-1}$, so the speed varies between 200 and 400 $\mathrm{km} \mathrm{s}^{-1}$ at the boundary. The fluctuation period is taken to be 180 days. This is somewhat larger than the observed largescale variations in ram pressure occurring on time scales of 
tens of days reported by Belcher et al. [1993], but it will be sufficient to demonstrate the essential physics involved in the response of the interaction to the fluctuations.

The fluctuations in ram pressure at the inner boundary ( 10 $\mathrm{AU})$ and at two other spatial locations upstream of the termination shock are shown in Figure 2. The ram pressure fluctuations at the inner boundary (10 $\mathrm{AU})$ are due to changes in just the flow speed, but those at the other two locations involve changes in both the flow speed and density. The fluctuations are shown tor only the first 2500 days of the simulation, but they remain unchanged for the total computation time of 7800 days. The termination shock in the equilibrium solution is located at about $80 \mathrm{AU}$; hence the fluctuations at $70 \mathrm{AU}$ are only $10 \mathrm{AU}$ upstream of the shock. The average value of the simulated ram pressure is less than the observed average value in Figure 3 of Belcher et al. [1993] owing to the lower-than-observed solar wind speed used in the simulations. This lower speed was used so that the termination shock would be located fairly close to the Sun when reasonable (anticipated) values were used for interstellar conditions. The magnitudes of the simulated ram pressure fluctuations in Figure 2 are at the low end of the observed magnitudes in Figure 3 of Belcher et al. [1993] and are similar to those of the fluctuations during solar minimum periods.

The temporal responses of the termination shock at the poles $\left(\theta=0^{\circ}, 180^{\circ}\right)$ and of the heliopause at the $0^{\circ}$ pole are shown in Figure 3 . The period of the response is the same as that of the applied speed oscillation (180 days). The amplitude of the response represents a total movement of the termination shock of about $1 \mathrm{AU}$. It should be noted that the amplitude of the response shown here is virtually identical to that when the applied flow speed oscillation amplitude at the inner boundary is either reduced to $75 \mathrm{~km} \mathrm{~s}^{-1}$ or increased to $125 \mathrm{~km} \mathrm{~s}^{-1}$. This indicates that the amplitude of the termination shock response may have saturated and that it would not increase significantly with further increases in the amplitude of the ram pressure fluctuations. If the flow speed oscillation amplitude is decreased to $50 \mathrm{~km} / \mathrm{s}$, there is a noticable reduction (about $20 \%$ ) in the oscillation amplitude. The oscillation amplitude at which the termination shock response saturates naturally depends on the oscillation period, but this dependence is not investigated here.

Although the simulated termination shock does not move as much as might be anticipated in response to the ram pressure fluctuations, the amplitudes of the spatial oscillations are significant, as shown in Figure 4. This figure compares the spatial structures of the density and radial flow speed along the poles in the dynamic equilibrium solution (solid curves) with the structures after the speed oscillation has been applied for 7800 days (dashed curves). It is evident from this figure that even though only a flow speed oscillation was applied at the inner boundary, large oscillations in both flow speed and density are generated in the solar wind. Notice from the plots along the $0^{\circ}$ pole that the fluctuations do not get through the heliopause into the interstellar medium. This is more apparent in Figure 5, in which the contour levels have been tailored to bring out fluctuations in 
the thermal pressure beyond the termination shock. The heliopause effectively acts as a barrier to contine the oscillations to the solar wind plasma.

The simulated response amplitude is substantially less than the $10 \mathrm{AU}$ (or larger) movement of the termination shock due to ram pressure fluctuations predicted by more approximate theories [Barnes, 1993; Suess, 1993]. It is also less than the shock movement estimated in the study by Belcher et al. [1993], in which the termination shock location was computed using observed ram pressure fluctuations with a $200 \mathrm{~km} \mathrm{~s}^{-1}$ limit on the shock speed. There are at least two reasons why the results of the studies cited above for the response amplitude of the termination shock differ from the present results.

First, the earlier studies are all one-dimensional and effectively assume that an isolated section of the termination shock responds independently of the rest of the termination shock. This, of course, does not happen in practice, and the entire termination shock responds collectively to solar wind ram pressure fluctuations. In the extreme case, one can envision situations in which one section of the termination shock is being subjected to a maximum in a ram pressure fluctuation while an adjoining section, not too far removed in angular distance from the first but at a different radial distance from the Sun, must respond to a minimum in the fluctuation. The first section will tend to move outward, and the second one will tend to move inward. Although this effect must be accommodated to a certain extent for all fluctuations, it becomes more important as the period of the fluctuations decreases. A second, much more important reason for the difference between the present results and those in earlier studies probably has to do with the more rapid response times either calculated or assumed in the earlier studies compared to those computed in the present study. This is now discussed in more detail.

\subsection{Termination shock response time}

As an estimate of the time it takes the termination shock to respond to changes in the solar wind quantities, we rapidly increase the solar wind flow speed at the inner boundary from $300 \mathrm{~km} \mathrm{~s}^{-1}$ in the equilibrium solution to $400 \mathrm{~km} \mathrm{~s}^{-1}$, hold the speed constant at $400 \mathrm{~km} \mathrm{~s}^{-1}$ at the inner boundary, and follow the evolution in time until the interaction approaches a new dynamic equilibrium. The flow speed is increased over 45 time cycles, which represent about 90 days, or half the period of the fluctuations used in the above study. The temporal response of the termination shock locarions at the poles and of the heliopause at the $0^{\circ}$ pole are given in Figure 6 . The termination shock and heliopause gradually approach their new equilibrium position.

The termination shock at the $0^{\circ}$ pole initially moves outward rapidly (between about 300 and 700 days in Figure 6) when it is first impacted by the solar wind disturbance. The shock at this location then rebounds (between about 700 and 1000 days) and then monotonically approaches an equilibrium position. The average outward speed of the termination shock along the $0^{\circ}$ pole during the initial rapid movement, approximately $73 \mathrm{~km} \mathrm{~s}^{-1}$, is followed by an 
average outward speed from 1000 to 6000 days of $7.2 \mathrm{~km}$ $\mathrm{s}^{-1}$. Both of these speeds are smaller than the maximum shock speed computed or estimated in earlier studies [Belcher et al., 1993; Barnes, 1993; Suess, 1993]. During the 90-day half-period of the fluctuation used above, the termination shock would move $3.8 \mathrm{AU}$ if traveling at $73 \mathrm{~km} \mathrm{~s}^{-1}$ and $0.38 \mathrm{AU}$ if traveling at $7.2 \mathrm{~km} \mathrm{~s}^{-1}$. These distances are smaller than the excursions predicted in the earlier studies and are, respectively, larger and smaller than the $1 \mathrm{AU}$ movement reported on above (section 3.2). The average speed of the termination shock during the computed $1 \mathrm{AU}$ fluctuations is about $20 \mathrm{~km} \mathrm{~s}^{-1}$ ( $1 \mathrm{AU}$ in 90 days). This study also shows that if a single increase in ram pressure of infinite duration is input, as Barnes [1993] did, the termination shock would then have time to reach larger speeds.

The termination shock at the $180^{\circ}$ pole also experiences an initial overshoot, but the heliopause does not. The motion of the termination shock along the $180^{\circ}$ pole following the initial overshoot in Figure 6 illustrates an important feature of the relaxation simulation. Once the downstream (from $90^{\circ}$ to $180^{\circ}$ ) portion of the termination shock reaches the vicinity of its equilibrium position, it oscillates over a very long period. This particular computation was not continued beyond the time shown in Figure 6 , so it is not known whether the oscillation amplitude decreases with time. The simulation to determine the initial equilibrium solution ( $\mathrm{sec}-$ tion 3.1) was continued long enough to determine that the oscillation amplitude was slowly decreasing with time. The spatial variation of the physical quantities and the streamlines for a $400 \mathrm{~km} \mathrm{~s}^{-1}$ solar wind speed are not shown here. However, other than the larger distances to the termination shock and the heliopause, there are no qualitative differences between that solution and the solution shown in Figure 1 for a $300 \mathrm{~km} \mathrm{~s}^{-1}$ solar wind speed.

\section{Discussion}

The response of the termination shock to large-scale fluctuations in the solar wind ram pressure is studied using numerical solutions of the gasdynamic equations. The first step in this study is to compute a dynamic equilibrium solution for the interaction between the solar wind and the interstellar medium by using a numerical relaxation procedure. The ram pressure fluctuations are then generated in the solar wind by temporal flow speed oscillations at the inner radial boundary for the computation. The subsequent solar wind ram pressure fluctuations are comparable in amplitude (near the observed minimum) and time scale (near the observed maximum) to those observed by Voyager 2 [Belcher et al., 1993].

The maximum movement of the termination shock in response to the ram pressure fluctuations is about $1 \mathrm{AU}$ for a $100 \mathrm{~km} \mathrm{~s}^{-1}$ flow speed oscillation at the inner boundary. Although only an oscillation in the flow speed is used as the driving mechanism, the solar wind disturbance consists of fluctuations in all physical quantities, including the density. When the driving flow speed oscillation is reduced to $75 \mathrm{~km}$ $\mathrm{s}^{-1}$, the termination shock response is almost identical to that 
for the larger-magnitude speed oscillations reported on here (which, in fact, is the same as that for $125 \mathrm{~km} \mathrm{~s}^{-1}$ oscillations), indicating that the response has saturated and would remain the same for even larger amplitude fluctuations. As a result, the fact that the magnitude of the ram pressure fluctuations are near the low end of those observed should not make any significant difference. That is, the termination shock response would be the same as that computed here even if the oscillation amplitude were increased to the maximum observed value. A reduction to $50 \mathrm{~km} \mathrm{~s}^{-1}$, however, reduces the termination shock movement somewhat. It should also be noted that the time scale for the fluctuations used here (180 days) is somewhat larger than observed. Consequently, the predicted 1-AU excursion should be considered an upper limit which would most likely be reduced for shorter-period fluctuations. One additional caution that should be mentioned is that the ambient or background solar wind flow speed of $300 \mathrm{~km} \mathrm{~s}^{-1}$ used in the study is lower than typical observed values. The effect of a larger ambient speed should be investigated, though one would anticipate that the deviation from the ambient value would have more of a role in determining the amplitude of the termination shock fluctuation.

The magnitude of the termination shock excursion computed here ( $1 \mathrm{AU})$ is substantially less than the 10 to 15 AU excursions predicted by other methods [Belcher et al., 1993; Barnes, 1993; Suess, 1993]. These other methods are more approximate in the sense that they are one-dimensional and thus consider only the movement of an isolated section of the termination shock rather than the collective response of the entire termination shock. The previous methods also do not consider the complete interaction region between the solar wind and the interstellar medium. One possible explanation for this difference is that the numerical differencing scheme used here contains inherent dissipation, as does any such numerical scheme, which may act to reduce the termination shock response time. This effect has been minimized in the present study by not adding any additional dissipation. Along the same lines, it should be emphasized that the differencing scheme is explicit, so all relevant time scales are included in the evolution.

The response of the termination shock may also be restricted by the fact that it is spread over several grid points in the simulation. This effect is difficult to quantify, but it should be noted that the spatial scale of the oscillations is considerably larger than the numerical width of the termination shock, which should tend to minimize the effect. A much more likely explanation for at least the major portion of the difference is that the termination shock simply does not respond as rapidly as either assumed or calculated in the earlier studies. The response time was simulated in the present study by computing the time needed for the interaction to reach a new equilibrium following a fixed change in the solar wind properties and was shown to be much larger than that used in the previous studies. However, given the numerical approximations and uncertainties, the actual termination shock response will probably be bounded by the values predicted here and those from the earlier work. 
Acknowledgments. Discussions with V. J. Pizzo and T. Holzer are gratefully appreciated. The careful and critical reading of the manuscript by $W$. Lewis resulted in a much improved version. The comments and suggestions by the two referees were also very helpful. This research was supported by NASA grant NAGW-2621.

The Editor thanks S.T. Suess and A. Barnes for their assistance in evaluating this paper. 


\section{References}

Baranov, V. B.. Gasdynamics of the solar wind interaction with the interstellar medium. Space Sci. Rev.. 52, 89, 1990.

Baranov. V. B.. and Y. G. Malama. The model of the solar wind interaction with the local interstellar medium. Numerical solution of self-consistent problem. J. Geophys. Res.. 98. 15,157, 1993.

Barnes. A., Distant solar wind plasma: View from the Pioneers. in Physics of the Outer Heliosphere, edited by S. Grzedzielski and D. E. Page, p. 235. Pergamon, New York. 1990.

Barnes, A., Motion of the heliospheric termination shock: A gas dynamic model, J. Geophys. Res., 98, 15,137, 1993.

Belcher, J. W., A. J. Lazarus, R. L. McNutt. Jr., and G. S. Gordon, Jr., Solar wind conditions in the outer heliosphere and the distance to the termination shock, J. Geophys. Res., 98, 15,177, 1993.

Book. D. L.. J. P. Boris, and K. J. Hain. Flux-corrected trans port, II. Generalization of the method. J. Comput. Phys., 18, 248, 1975.

Davis, L. E., Jr., Interplanetary magnetic fields and cosmic rays, Phys. Rev., 100. 1440. 1955.

Donohue. D. J., and G. P. Zank. Steady state and dynamical structure of a cosmic-ray-modified termination shock. $J$. Geophys. Res., 98, 19,005, 1993.

Holzer, T. E., Interaction between the solar wind and the interstellar medium, Annu. Rev. Astron. Astrophys., 27. 199. 1989.

Lapidus, A., A detached shock calculation by second-order finite differences. J. Comput. Phys., 2, 154, 1967.

Parker, E. N., The stellar-wind regions, Astrophys. J., 134, 20, 1961.

Richtmyer, R. D., and K. W. Morton, Difference Methods for Initial Value Problems, p. 300, Interscience, New York, 1967.

Steinolfson, R. S., V. J. Pizzo, and T. Holzer, Gasdynamic models of the solar wind/interstellar medium interaction, Geophys. Res. Lett.. 21, 245, 1994.

Suess, S. T., The heliopause, Rev. Geophys., 28, 97, 1990.

Suess, S. T., Temporal variations in the termination shock distance, J. Geophys. Res., 98, 15, 147, 1993.

R.S. Steinolfson. Southwest Research Institute, 6220 Culebra Road, P.O. Drawer 28510. San Antonio, TX 78238-5166.

(Received January 13, 1994; revised March 7, 1994; accepted March 10, 1994.)

Copyright 1994 by the American Geophysical Union.

Paper number 94JA006770

0148-0227/94/94JA-00677\$05.00

STEINOLFSON: TERMINATION SHOCK AND SOLAR WIND FLUCTUATIONS

STEINOLFSON: TERMNATION SHOCK AND SOLAR WND FLUCTUATIONS

STEINOLFSON: TERMINATTON SHOCK AND SOLAR WIND FLUCTUATIONS

STEINOLFSON: TERMINATION SHOCK AND SOLAR WIND FLUCTUATIONS

STENOLFSON: TERMINATION SHOCK AND SOLAR WIND FLUCTUATIONS

STEINOLFSON: TERMINATION SHOCK AND SOLAR WIND FLUCTUATIONS 
STEINOLFSON: TERMINATION SHOCK AND SOLAR WIND FLUCTUATIONS

STEINOLFSON: TERMINATION SHOCK AND SOLAR WIND FLUCTUATIONS

STEINOLFSON: TERMINATION SHOCK AND SOLAR WIND FLUCTUATIONS

STEINOLFSON: TERMINATION SHOCK AND SOLAR WIND FLUCTUATIONS

STEINOLFSON: TERMINATION SHOCK AND SOLAR WIND FLUCTUATIONS

STEINOLFSON: TERMINATION SHOCK AND SOLAR WIND FLUCTUATIONS

STEINOLFSON: TERMINATION SHOCK AND SOLAR WIND FLUCTUATIONS

STEINOLFSON: TERMINATION SHOCK AND SOLAR WIND FLUCTUATIONS

STEINOLFSON: TERMINATION SHOCK AND SOLAR WIND FLUCTUATIONS

STEINOLFSON: TERMINATION SHOCK AND SOLAR WIND FLUCTUATIONS

STEINOLFSON: TERMINATION SHOCK AND SOLAR WIND FLUCTUATIONS

STEINOLFSON: TERMINATION SHOCK AND SOLAR WIND FLUCTUATIONS

Figure 1. Thermodynamic quantities and velocity streamlines in the relaxed dynamic equilibrium solution. The radial range extends from the inner computation boundary at 10 $\mathrm{AU}$ to $400 \mathrm{AU}$. The interstellar flow (relative to the solar system) is from left to right. The termination shock location drawn on the streamline plot was determined from the contour plots of the thermodynamic variables. For the thermodynamic quantities, the value used to construct the plots is $\left(Q \cdot Q_{\text {is }}\right) / Q_{\text {is, }}$, where $Q_{\text {is }}$ represents the interstellar value. A contour level of zero represents the interstellar value, and values greater (smaller) than the interstellar value are represented by solid (dashed) contours. The density contours range from -0.96 to 0.24 in increments of 0.08 , pressure ranges from -0.8 to 0.5 in increments of 0.1 , and temperature ranges from 10 to 100 in increments of 10 .

Figure 2. Temporal fluctuations in the solar wind ram pressure at three locations along the pole at $\theta=0^{\circ}$ upstream of the termination shock. The fluctuations are shown for only the first 2500 days but remain unchanged throughout the remainder of the simulation.

Figure 3. Temporal fluctuations in the locations of the termination shock along the poles at $\theta=0^{\circ}$ and $\theta=180^{\circ}$ and of the heliopause along the pole at $\theta=0^{\circ}$. The fluctuations are shown for only the tirst 2500 days but remain unchanged throughout the remainder of the simulation.

Figure 4. Spatial fluctuations in the density and flow speed along the poles (at $\theta=0^{\circ}, 180^{\circ}$ ) at the end of the simulation (dashed curves) superimposed on the dynamic equilibrium state (solid curves).

Figure 5. Contour plots of the thermal pressure illustrating confinement of the fluctuations to the heliosphere. The heavy dashed curve marks the location of the heliopause. The fluctuations within the termination shock do not appear in this figure, since the pressure within the termination shock is below the minimum contour level. 
Figure 6. Temporal responses of the termination shock locations at the poles and of the heliopause at the $0^{\circ}$ pole as the solar wind speed in the dynamic equilibrium solution in Figure 1 is increased over 90 days from $300 \mathrm{~km} \mathrm{~s}^{-1}$ to 400 $\mathrm{km} \mathrm{s}^{-1}$ and then at that level.

Figure 1. Thermodynamic quantities and velocity streamlines in the relaxed dynamic equilibrium solution. The radial range extends from the inner computation boundary at $10 \mathrm{AU}$ to $400 \mathrm{AU}$. The interstellar flow (relative to the solar system) is from left to right. The termination shock location drawn on the streamline plot was determined from the contour plots of the thermodynamic variables. For the thermodynamic quantities, the value used to construct the plots is $\left(Q-Q_{i s}\right) / Q_{i s}$, where $Q_{i \text { is }}$ represents the interstellar value. A contour level of zero represents the interstellar value, and values greater (smaller) than the interstellar value are represented by solid (dashed) contours. The density contours range from -0.96 to 0.24 in increments of 0.08 , pressure ranges from -0.8 to 0.5 in increments of 0.1 , and temperature ranges from 10 to 100 in increments of 10 .

Figure 2. Temporal fluctuations in the solar wind ram pressure at three locations along the pole at $\theta=0^{\circ}$ upstream of the termination shock. The fluctuations are shown for only the first 2500 days but remain unchanged throughout the remainder of the simulation.

Figure 3. Temporal fluctuations in the locations of the termination shock along the poles at $\theta=0^{\circ}$ and $\theta=180^{\circ}$ and of the heliopause along the pole at $\theta=0^{\circ}$. The fluctuations are shown for only the first 2500 days but remain unchanged throughout the remainder of the simulation.

Figure 4. Spatial fluctuations in the density and flow speed along the poles (at $\theta=0^{\circ}, 180^{\circ}$ ) at the end of the simulation (dashed curves) superimposed on the dynamic equilibrium state (solid curves).

Figure 5. Contour plots of the thermal pressure illustrating confinement of the fluctuations to the heliosphere. The heavy dashed curve marks the location of the heliopause. The fluctuations within the termination shock do not appear in this figure, since the pressure within the termination shock is below the minimum contour level.

Figure 6. Temporal responses of the termination shock locations at the poles and of the heliopause at the $0^{\circ}$ pole as the solar wind speed in the dynamic equilibrium solution in Figure 1 is increased over 90 days from $300 \mathrm{~km} \mathrm{~s}^{-1}$ to $400 \mathrm{~km} \mathrm{~s}^{-1}$ and then at that level. 

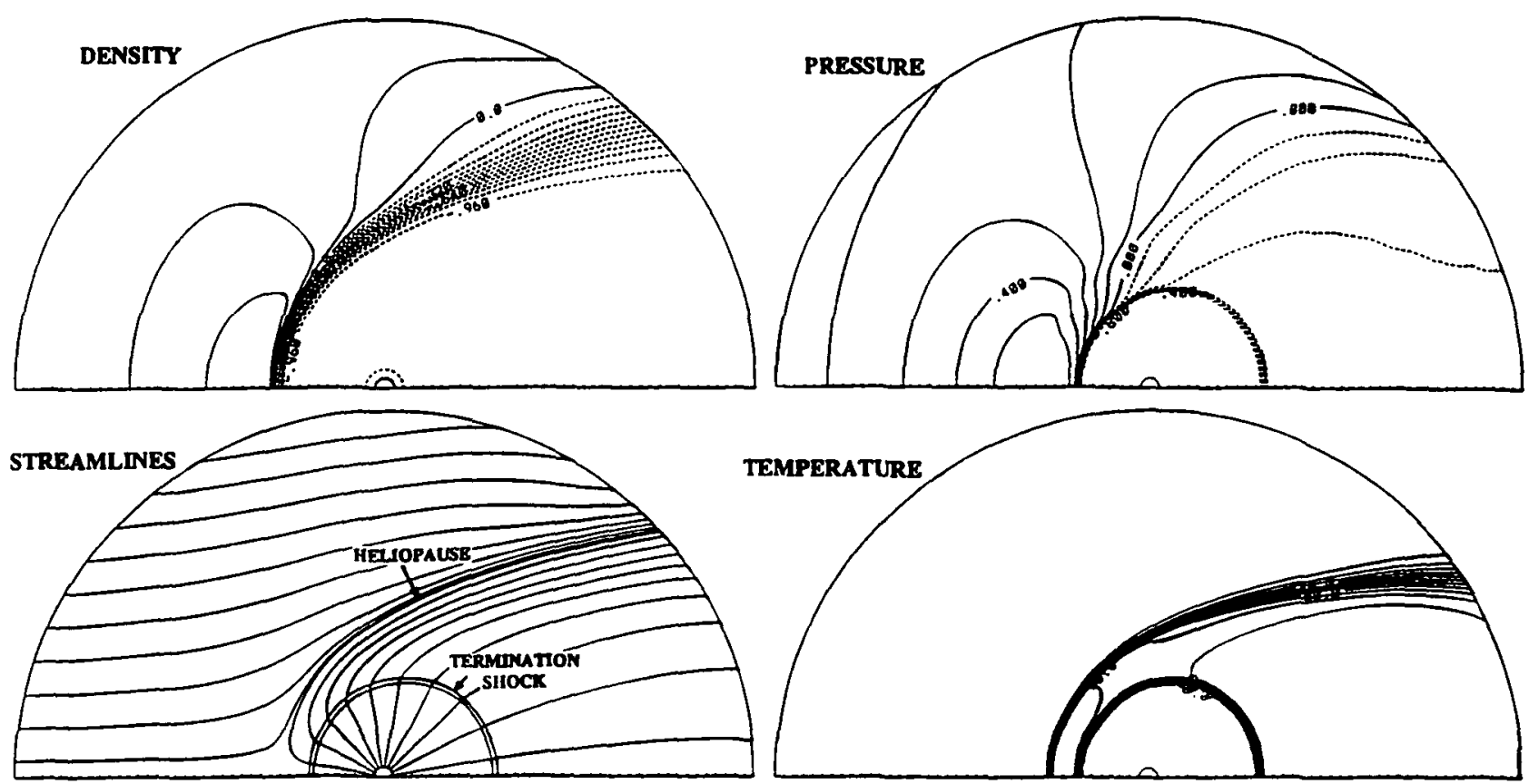

Figure 1. 


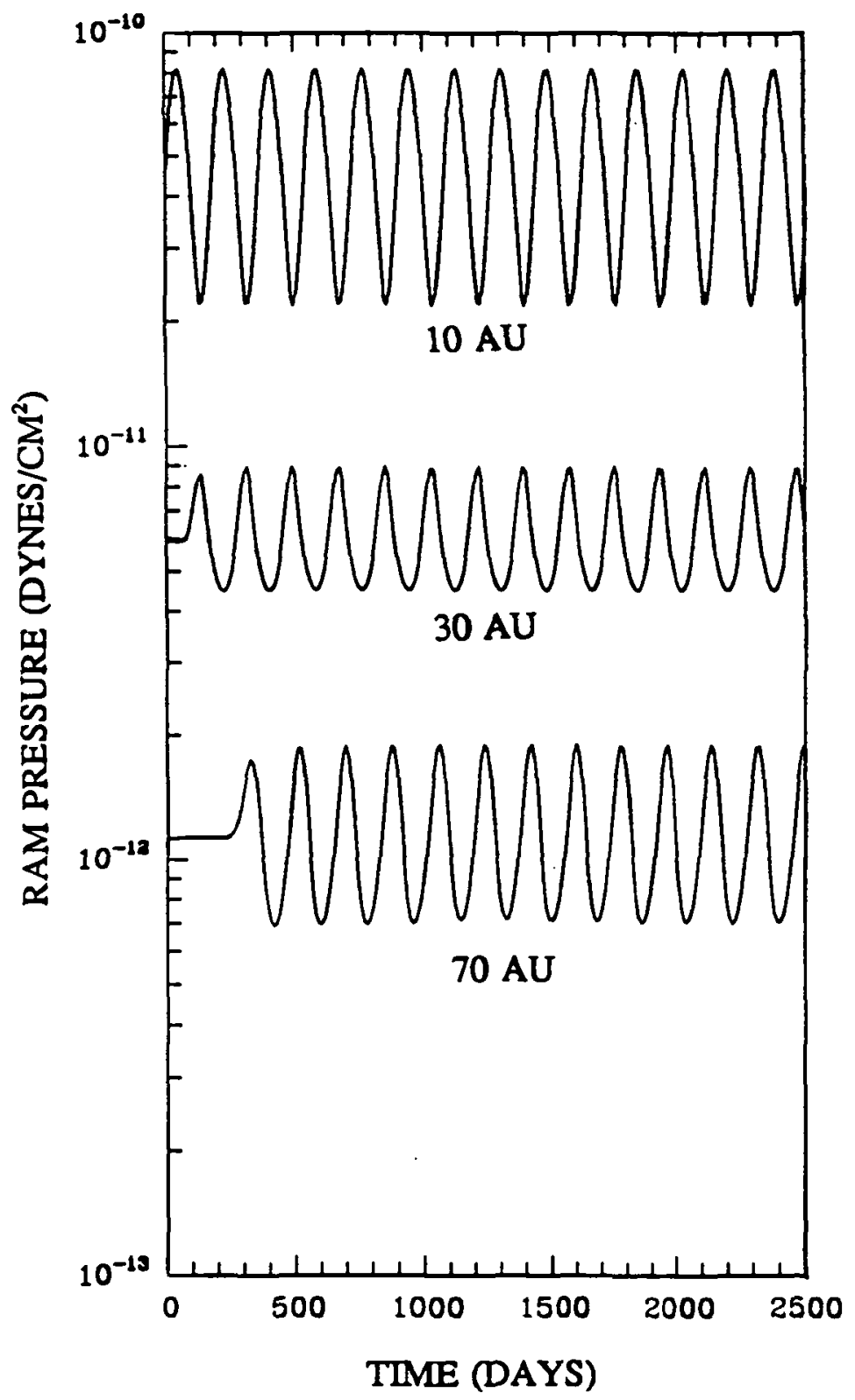

Figure 2. 


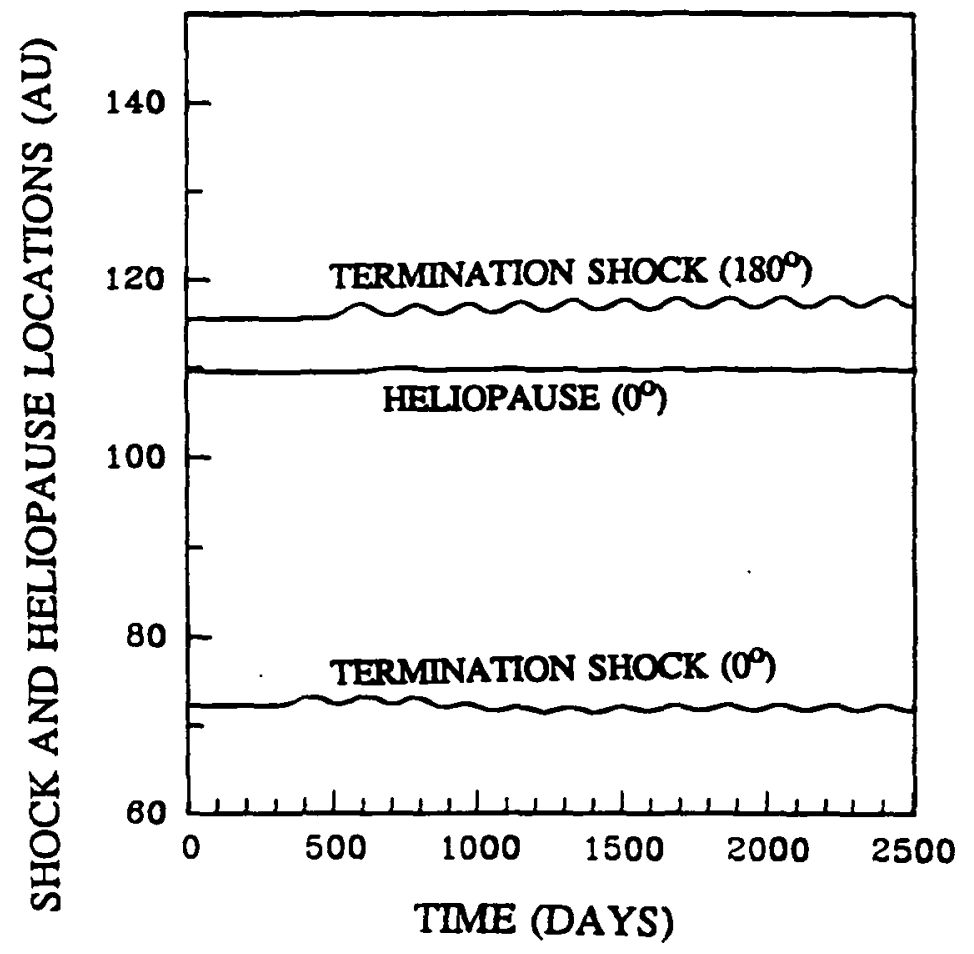

Figure 3. 

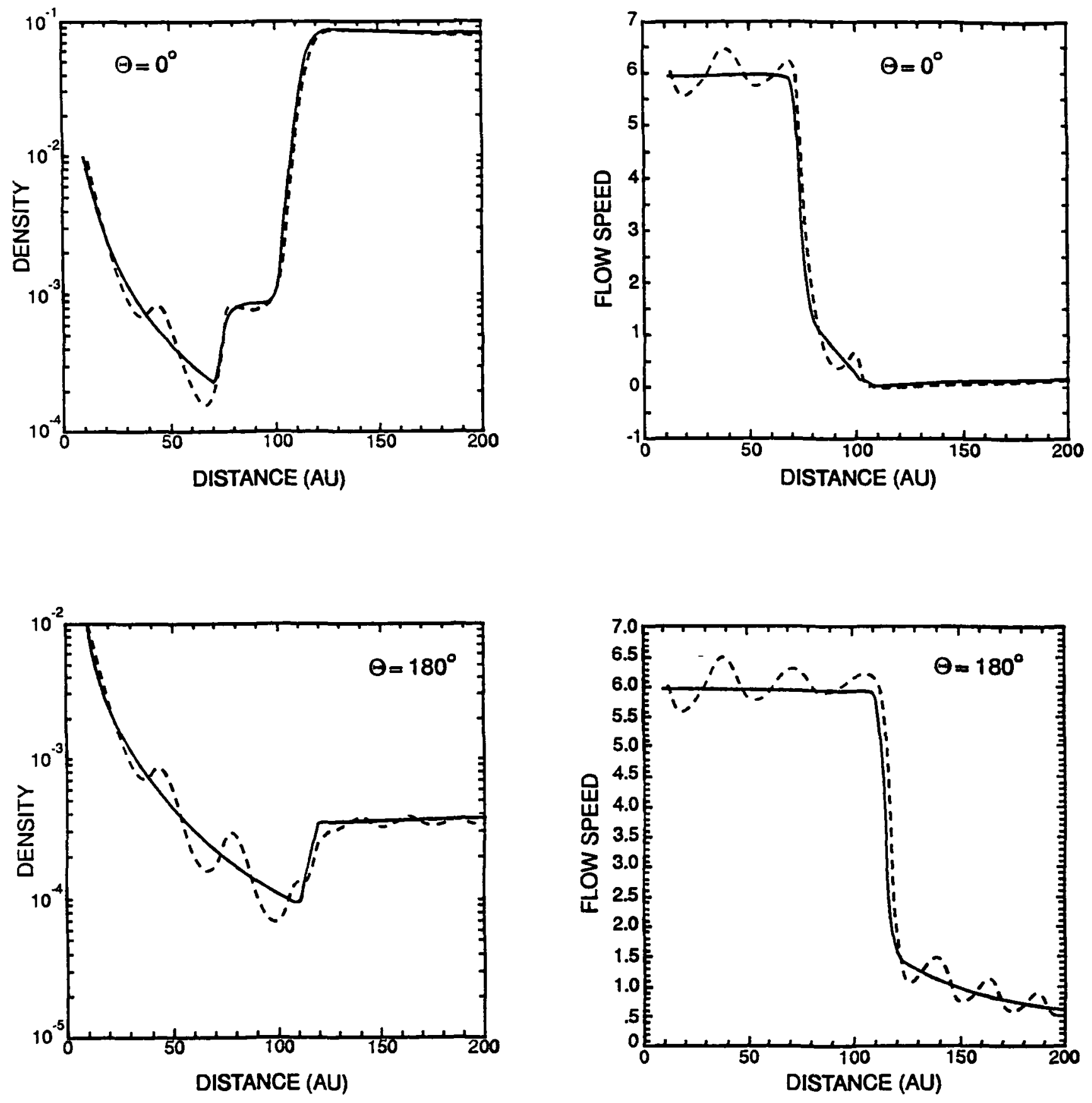

Figure 4. 


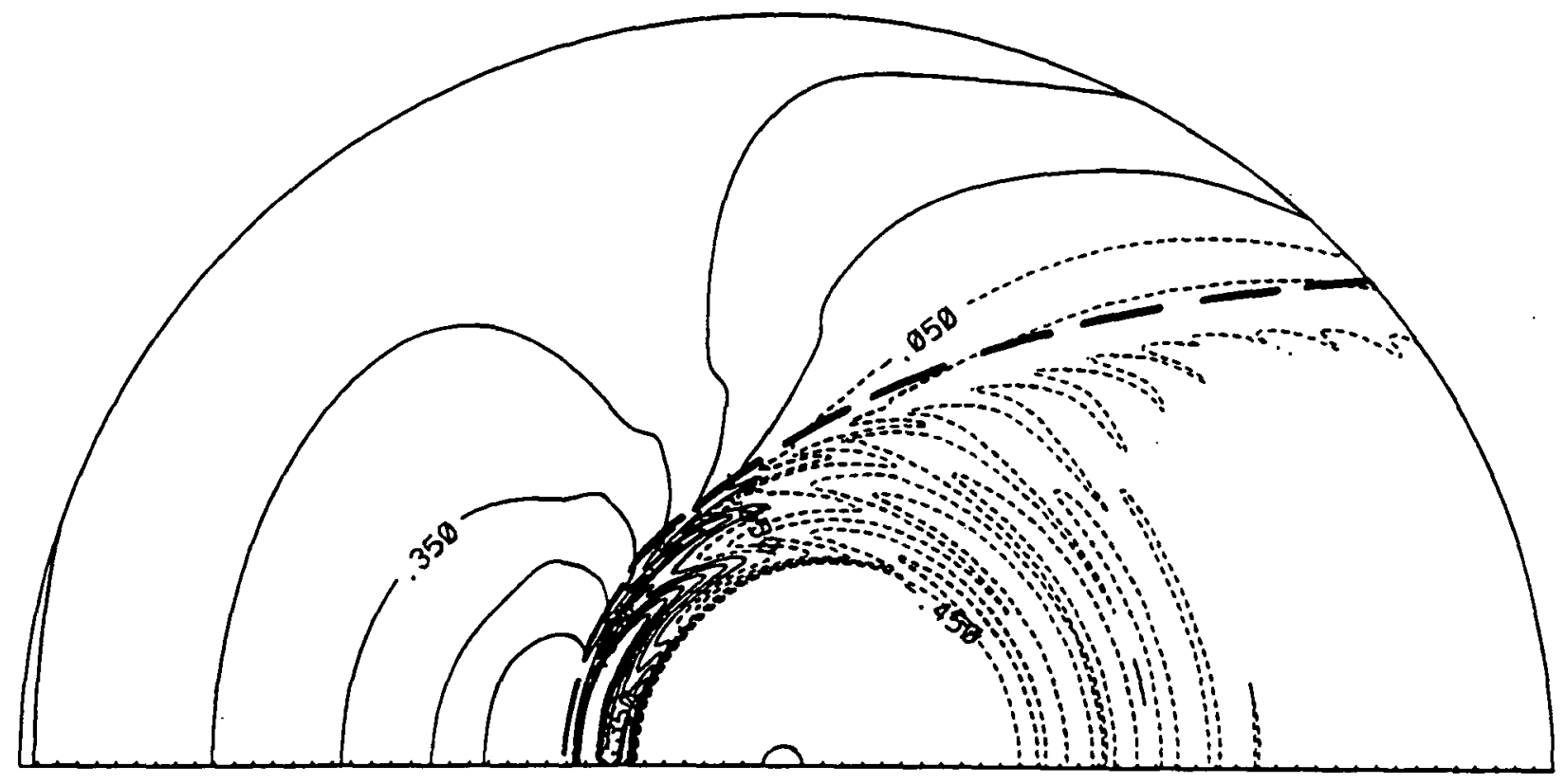

Figure 5. 


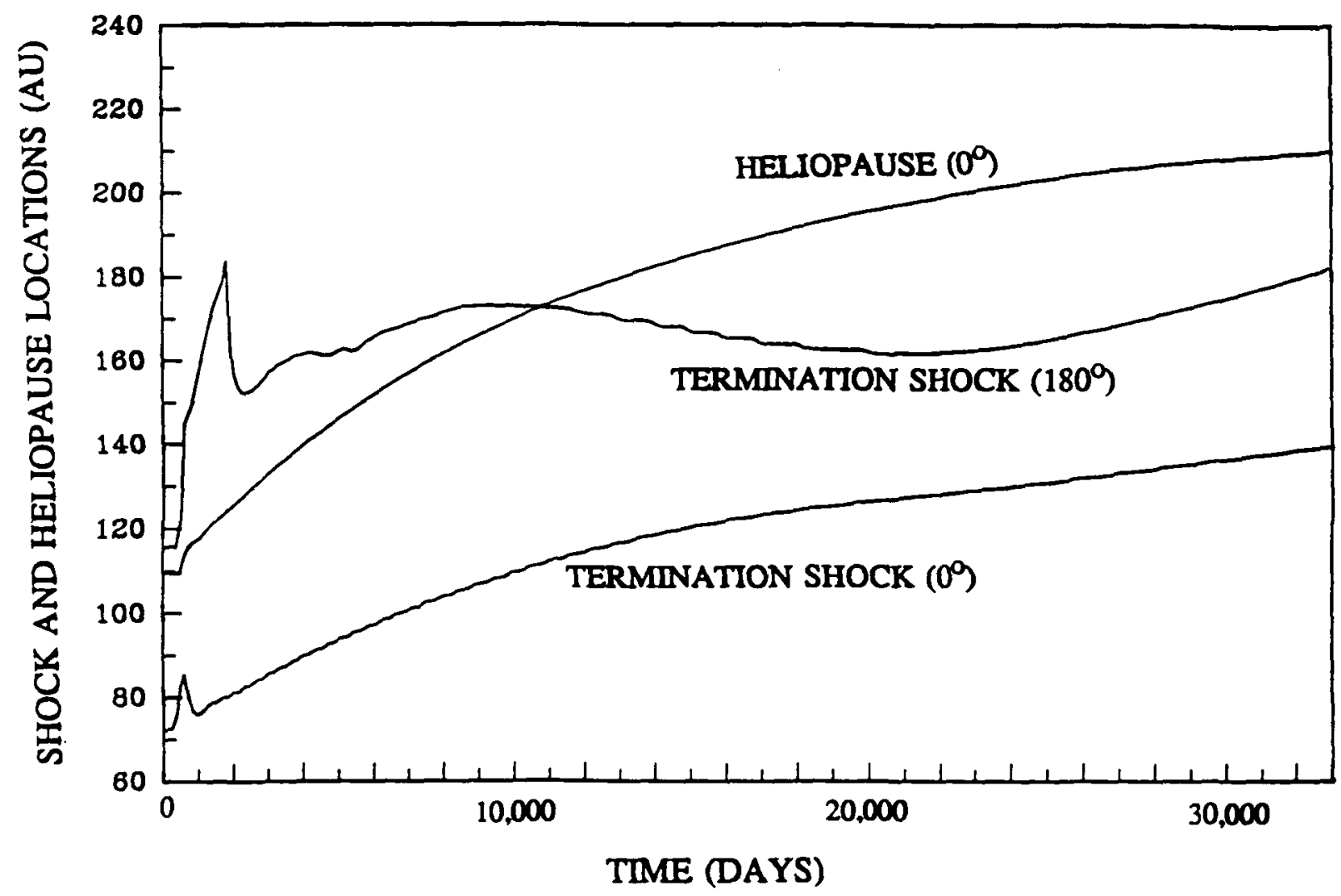

Figure 6. 